diseases perspective, the only thing that is standardized are the outpatients, and they are probably $50 \%$ of the volume."

This was also true for patients undergoing cardiac surgery in the STOP SSI study (cited by Ariyo et al) ${ }^{2}$ and similar subsequent unpublished studies. In the STOP SSI study, the significant reduction in complex $S$. aureus SSIs was only seen among orthopedic surgery patients but not cardiac surgery patients. The orthopedic surgery patients tended to be scheduled for elective surgery and had outpatient preoperative clinic visits in the 30 days prior to surgery. During this visit, the orthopedic patients were provided chlorhexidine body wash, were screened for $S$. aureus colonization and, if positive, were provided with a 5-day supply of mupirocin nasal ointment. In contrast, the cardiac surgery patients did not have a standardized outpatient preoperative clinic appointment and were often only seen in the inpatient setting.

Similarly, only $1.6 \%$ of patients undergoing urgent/emergent operations were fully adherent to the STOP SSI bundle, $40 \%$ of patients undergoing scheduled operations were fully adherent. This factor was reflected in the outcomes, in which there was only a statistically significant reduction in complex $S$. aureus SSIs among the scheduled operations and not the urgent/emergent operations. In a similar study performed in Veterans Affairs (VA) hospitals, a cardiac case manager from a small VA hospital stated:

"[The patient] could have a scheduled outpatient [cardiac catheterization] here and end up on a balloon pump, and then [be sent] via ambulance to the University for [urgent] open heart surgery. Those are not going to be ones we catch, obviously."

Many infection prevention interventions, such as chlorhexidine washes and MRSA nasal screening and decolonization, require significant upfront investment of time and resources. Thus, providing these services to less stable patients and to add-on patients is difficult, if not impossible. Environmental barriers do not allow for showering and time restrictions do not allow for MRSA screening and multiple-day decolonization regimens.

These implementation challenges are critical areas of future research. First, as a field, we need better ways to implement infection prevention interventions and to track which patients receive them and which do not. In addition to healthcareassociated infection outcomes, consideration of intermediate, implementation outcomes (eg, adherence to the intervention), should be included in assessments of efficacy and effectiveness, so that the potential for confounding by indication can be assessed and addressed. Second, we need to find better ways to triage infection prevention strategies. Under the current system, the most aggressive care is being targeted to the lowest-risk patients. Infection prevention interventions that can be rapidly implemented in the day-of-surgery area or the operating room could be more effective than interventions that need to be started multiple days before surgery. More research is needed to determine how we can improve implementation and overcome barriers for the most vulnerable patients, rather than targeting care where it is most convenient to do so.

Author ORCIDs. Westyn Branch-Elliman, Marin Schweizer, (iD) 0000-0002-3604-0093

0000-0002-9658-5124,

Acknowledgments. The authors would like to thank Heather Schacht Reisinger, Cassie Goedken, and Stacey Hockett-Sherlock for their work performing qualitative interviews.

Financial support. Funding for this letter was provided by the National Institutes of Health (grant no. NHLBI 1K12HL138049-01) and the Agency for Healthcare Research and Quality (AHRQ grant nos. HHSA2902006100021I, HS022467-02; VA HRS\&D CREATE CRE 12-291).

Conflicts of interest. M.S. received grant funding from PDI Healthcare.

\section{References}

1. Ariyo P, Zayed B, Riese V, et al. Implementation strategies to reduce surgical site infections: a systematic review. Infect Control Hosp Epidemiol 2019:1-14.

2. Schweizer ML, Chiang HY, Septimus E, et al. Association of a bundled intervention with surgical site infections among patients undergoing cardiac, hip, or knee surgery. JAMA 2015;313:2162-2171.

\title{
Rapid and economical detection of eight carbapenem-resistance genes in Enterobacteriaceae, Pseudomonas spp, and Acinetobacter spp directly from positive blood cultures using an internally controlled multiplex-PCR assay
}

\author{
Surojit Das MSc, $\mathrm{PhD}^{1}$, Subhanita Roy $\mathrm{MSc}^{1}$, Samadrita Roy $\mathrm{MSc}^{1}$, Gaurav Goel MD, $\mathrm{DNB}^{1}$, Kamini Walia PhD, MPH${ }^{2}$, \\ Sudipta Mukherjee MD, IDCCM, FNB, EDICM ${ }^{3}$, Sanjay Bhattacharya MD, DNB, FRCPath ${ }^{1}(\mathbb{0})$ and Mammen Chandy MD \\ FRCPA, FRACP, FRCP ${ }^{4}$
}

${ }^{1}$ Department of Microbiology, Tata Medical Center, Kolkata, India, ${ }^{2}$ Indian Council of Medical Research, New Delhi, India, ${ }^{3}$ Department of Critical Care, Tata Medical Center, Kolkata, India and ${ }^{4}$ Department of Clinical Hematology, Tata Medical Center, Kolkata, India

\footnotetext{
Author for correspondence: Dr Sanjay Bhattacharya, Emails: sanjay.bhattacharya@ tmckolkata.com or drsanjay1970@hotmail.com

Cite this article: Das S, et al. (2019). Rapid and economical detection of eight carbapenem-resistance genes in Enterobacteriaceae, Pseudomonas spp, and Acinetobacter spp directly from positive blood cultures using an internally controlled multiplex-PCR assay. Infection Control \& Hospital Epidemiology, 40: 737-739, https://doi.org/10.1017/ ice.2019.79
}

To the Editor-The World Health Organization (WHO) has recognized carbapenem-resistant Enterobacteriaceae (CRE), Pseudomonas aeruginosa (CRPsA), and Acinetobacter baumannii (CRAB) as critical pathogens that cause significant morbidity and mortality in patients with bloodstream infections (BSIs), 
Table 1. Distribution of Different Carbapenemases in Enterobacteriaceae, Pseudomonas spp, and Acinetobacter spp Isolated From Blood Samples

\begin{tabular}{|c|c|c|c|c|c|c|c|c|c|c|c|c|}
\hline \multirow[b]{2}{*}{ Organism (n) } & \multicolumn{6}{|c|}{ Single Carbapenemase } & \multicolumn{6}{|c|}{ Double Carbapenemase } \\
\hline & NDM & OXA-48 & KPC & VIM & OXA-23 & OXA-58 & NDM/ OXA-48 & NDM/OXA-58 & $\mathrm{KPC} / \mathrm{VIM}$ & NDM/OXA-23 & NDM/VIM & OXA-48/OXA-58 \\
\hline E. coli (32) & 25 & 3 & ND & ND & ND & ND & 4 & ND & ND & ND & ND & ND \\
\hline K. pneumoniae (82) & 2 & 62 & 2 & ND & ND & ND & 16 & ND & ND & ND & ND & ND \\
\hline K. oxytoca (1) & 1 & ND & ND & ND & ND & ND & ND & ND & ND & ND & ND & ND \\
\hline E. cloacae (7) & 6 & 1 & ND & ND & ND & ND & ND & ND & ND & ND & ND & ND \\
\hline Citrobacter freundii (1) & 1 & ND & ND & ND & ND & ND & ND & ND & ND & ND & ND & ND \\
\hline A. baumannii (13) & 2 & ND & ND & ND & 4 & 2 & ND & 3 & ND & 2 & ND & ND \\
\hline Acinetobacter spp (2) & 1 & ND & ND & ND & ND & ND & ND & 1 & ND & ND & ND & ND \\
\hline P. aeruginosa (15) & 11 & ND & ND & 1 & ND & ND & ND & ND & 2 & ND & 1 & ND \\
\hline Pseudomonas spp (1) & ND & ND & ND & 1 & ND & ND & ND & ND & ND & ND & ND & ND \\
\hline
\end{tabular}

Note: ND, not detected by polymerase chain reaction assay.

especially in healthcare settings. ${ }^{1,2}$ Carbapenem resistance challenges empirical and targeted antibiotic treatment involving $\beta$ lactam antibiotics, including carbapenems, and it results in expensive and complicated alternative anti-infective strategies with polymyxin-B, colistin, tigecycline, and fosfomycin. ${ }^{3}$ Despite their high cost ( US\$62 per test), commercially available PCR-based tests have been used to screen clinically important carbapenemases, known as the "Big 5 " genes: metallo- $\beta$-lactamases (MBLs) (ie, NDM, IMP, and VIM), KPC, and the OXA48 family. These tests use rectal swab samples with short turnaround times (TATs), which contribute significantly to infection control efforts. ${ }^{2,4,5}$ Few validated tests are available to meet the acute need in BSI cases. ${ }^{2,4} \mathrm{~A}$ rapid, inexpensive, and accessible testing tool for carbapenemase producing-carbapenem resistant organisms (CP-CRO) as pathogens of BSI is of paramount importance for early institution of effective antimicrobial therapy and infection prevention and its control. In the present study, a predesigned multiplex PCR assay was validated that targets the OXA carbapenemases (OXA-23, OXA-24/-143, and the OXA-58 family) commonly harbored by Acinetobacter spp, as well as with the "Big 5 " genes, in DNA extracts of gram-negative bacilli positive blood-culture (BC) broths.

In this prospective study, consecutive $\mathrm{BC}$ broth samples $(\mathrm{n}=415)$ were collected from patients $(\mathrm{n}=278)$ with laboratory-confirmed BSIs at Tata Medical Center, Kolkata, India, from July 2017 to September 2018. Blood culturing was performed using the BacT/Alert 3D system (bioMérieux, Marcy-l'Étoile, France), and identification and susceptibility testing of $\mathrm{BC}$ isolates were performed using the Vitek2 system (bioMérieux). Carbapenem susceptibility results were interpreted following Clinical Laboratory Standard Institute (CLSI) guidelines. ${ }^{6}$

DNA was extracted from positive BC broths using the alkali wash and heat lysis method. ${ }^{7}$ Additionally, $20 \mu \mathrm{L}$ McFarland 3 turbidity standard of A. baumannii ATCC19606 was added to $500 \mu \mathrm{L}$ $\mathrm{BC}$ broth before extraction as an internal extraction control (IEC). Two multiplex PCRs were then performed to detect 8 carbapenemase genes (as described earlier) with a modification: CARBA (KPC, 353 base pairs [bp]; NDM, 603 bp; VIM, 437 bp; IMP, 387 bp; OXA-48 family, $265 \mathrm{bp}$ ) and OXA (OXA-23 family, $330 \mathrm{bp}$; OXA24/143 family, 271 bp; OXA-58 family, 688 bp; with Acinetobacterderived AmpC [ADC] as the IEC and PCR control, 1,059 bp). ${ }^{8}$ The PCR amplicons were visualized on $3 \%$ prestained agarose gels. Bidirectional sequencing was performed using a 3500 DNA analyzer
(Applied Biosystems, Foster City, CA) for confirmation. Analytical specificities of multiplex PCRs were validated using bacterial and fungal reference strains and clinical isolates. For assay standardization, cultured reference strains were suspended in normal saline to a density of $0.5 \mathrm{McF}$ arland turbidity standard $\left(\sim 1.5 \times 10^{8} \mathrm{CFU} / \mathrm{mL}\right)$ using a Densimat (bioMerieux) followed by preparation of 10 -fold serial dilutions ranging from $10^{7}$ to $10^{2} \mathrm{CFU} / \mathrm{mL}$. Known negative BC samples $(450 \mu \mathrm{L})$ incubated for 5 days were spiked with bacterial dilutions $(50 \mu \mathrm{L})$, and DNA extraction followed by PCR was preformed to check analytical sensitivity.

Statistical performance indicators of the multiplex PCRs were determined using MedCalc (MedCalc Software, Mariakerke, Belgium) using carbapenem susceptibilities of Enterobacteriaceae, Pseudomonas spp, and Acinetobacter spp (EPA) as references. Non-EPA, intrinsically CRO, and uncultivable organisms were considered negative controls. Interrater agreement using Cohen's $\kappa$ was used to measure the concordance between culture and multiplex PCRs.

Of 153 CARBA-OXA multiplex PCR-positive samples, 122 had a single target (NDM, 48; OXA-48 family, 66; KPC, 2; VIM, 2; and OXA-23 family, 4) and 31 had double targets (NDM/OXA-48 family, 20; NDM/OXA-58 family, 5; KPC/VIM, 2; NDM/OXA23 family, 2; NDM/VIM, 1; and OXA-48/OXA-58 family, 1) (Table 1). The analytical specificities of the PCRs were $100 \%$ for detecting the 8 carbapenemases; the sensitivities ranged between 500 and $5000 \mathrm{CFU} / \mathrm{mL}$ for different carbapenemases using spiked BC samples. Among 415 BC samples, accuracy of the assay was marginally improved $(95.9 \%$ vs $96.9 \%)$ using both the "Big 5 " CARBA and extended OXA panels (Supplementary Table 1 online). Discrepant results between the culture method and multiplex-PCRs were observed in 13 samples. The TAT for the detection of carbapenemases was $<4$ hours using BC positive broth, including DNA extraction ( $\sim 50$ minutes), PCR amplification $(\sim 120$ minutes), and agarose-gel electrophoresis and documentation ( $\sim 30$ minutes). The cost of consumables and reagents for running the assays was only $\sim$ US $\$ 10$ per sample.

Early and rapid detection of carbapenemases in common gramnegative bacilli is of paramount importance for optimizing antimicrobial therapy, as a tool for antimicrobial stewardship programs, for hospital infection control, and for improving patient outcomes with regard to morbidity, mortality, reducing length of hospitalization, and minimizing the cost of health care. Compared with commercial assays (eg, Carba R GeneXpert from Cepheid, 
Sunnyvale, CA), the cost of the current assay was significantly less with a convenient TAT (same-day result) using this noncommercial DNA extraction method and end-point multiplex PCR format. ${ }^{4,5,9}$ In this study, we validated the diagnostic performance of internally controlled multiplex-PCRs targeting 8 carbapenemases in BC-positive samples. In the wake of increased carbapenem-based antimicrobial therapies, multiplex PCR may prove to be a useful tool for detecting carbapenem resistance and thus facilitating early infection control actions in clinical settings. The strengths of the current study include (1) demonstration of the molecular epidemiology of carbapenem resistance genes among a group of oncology patients in eastern India, (2) development of a rapid and cost effect test suitable for implementation in resource constrained settings; (3) application of molecular tests for gram negative bacteremia to optimize antibiotic therapy; (4) identification of potential targets for developing new drugs to treat NDM and OXA positive GNB infections, and (5) use of OXA-23/24/-58 PCRs, which has been rarely investigated for diagnostic purposes apart from its importance as a marker in CRAB associated with outbreaks (OXA-23). ${ }^{5}$ The implementation of this new, low-cost tool in resource-limited settings may enable better management of gram-negative sepsis. ${ }^{10}$

Author ORCIDs. Sanjay Bhattacharya, (iD 0000-0003-4139-1039

Supplementary material. To view supplementary material for this article, please visit https://doi.org/10.1017/ice.2019.79.

Acknowledgments. We would like to thank the Indian Council of Medical Research for supporting the study.

Financial support. The financial support for this work came from the Indian Council of Medical Research.
Conflicts of interest. All authors report no conflicts of interest relevant to this article.

\section{References}

1. WHO. Global priority list of antibiotic-resistant bacteria to guide research, discovery, and development of new antibiotics. WHO documents. Geneva: World Health Organization; 2017.

2. Banerjee R, Humphries R. Clinical and laboratory considerations for the rapid detection of carbapenem-resistant Enterobacteriaceae. Virulence 2017;8:427-439.

3. Cortegiani A, Russotto V, Graziano G, et al. Use of Cepheid Xpert Carba-R1 for rapid detection of carbapenemase-producing bacteria in abdominal septic patients admitted to intensive care unit. PLoS One 2016;11:e0160643.

4. Findlay J, Hopkins KL, Meunier D, Woodford N. Evaluation of three commercial assays for rapid detection of genes encoding clinically relevant carbapenemases in cultured bacteria. J Antimicrob Chemother 2015;70:1338-1342.

5. Codjoe FS, Donkor ES. Carbapenem resistance: a review. Med Sci 2018; 6:1-28.

6. CLSI. Performance Standards for Antimicrobial Susceptibility Testing, 28th edition, CLSI supplement M100. Wayne, PA: Clinical and Laboratory Standards Institute; 2017.

7. Millar BC, Jiru X, Moore JE, Earle JA. A simple and sensitive method to extract bacterial, yeast and fungal DNA from blood culture material. J Microbial Methods 2000;42:139-147.

8. Bogaerts P, Rezende de Castro R, de Mendonca R, Haung TD, Denis O, Glupczynski Y. Validation of carbapenemase and extended-spectrum $\beta$-lactamase multiplex endpoint PCR assays according to ISO 15189. J Antimicrob Chemother 2013;68:1576-1582.

9. Tato M, Ruiz-Garbajosa P, Traczewski M, et al. Multisite evaluation of Cepheid Xpert Carba-R assay for detection of carbapenemaseproducing organisms in rectal swabs. J Clin Microbiol 2016;54: 1814-1819.

10. Nordmann P, Poirel L. The difficult-to-control spread of carbapenemase producers among Enterobacteriaceae worldwide. Clin Microbial Infect 2014;20:821-830.

\title{
Impact of biofilm production on polymyxin B susceptibility among Pseudomonas aeruginosa clinical isolates
}

\author{
Tiele da Silva Carvalho Pharm and Leandro Reus Rodrigues Perez PhD (1) \\ Curso de Especialização em Microbiologia Clínica, Universidade Federal do Rio Grande do Sul, Porto Alegre, Brazil
}

To the Editor-Polymyxin B is an old class of nonribosomal cyclic lipopeptide antibiotics that has been used for the treatment of gram-negative bacterial infection such as multidrug-resistant Pseudomonas aeruginosa, especially carbapenem-resistant isolates. ${ }^{1}$

Bacteria are usually able to evolve different strategies to sense, respond, and adapt to bactericidal agents including polymyxins. Although biofilm is a well-established response strategy to an antimicrobial agent, little is known about the impact of biofilms produced by $P$. aeruginosa regarding polymyxin susceptibility. ${ }^{2}$

Biofilms - designated as an aggregation of bacterial cells-are crucially important due to a high adhesion ability on surfaces

\footnotetext{
Author for correspondence: Leandro Reus Rodrigues Perez, Email: leandro.reus@gmail. com

Cite this article: da Silva Carvalho T and Rodrigues Perez LR. (2019). Impact of biofilm production on polymyxin B susceptibility among Pseudomonas aeruginosa clinical isolates. Infection Control \& Hospital Epidemiology, 40: 739-740, https://doi.org/10.1017/ice.2019.85

and a worrying ability to withstand high concentrations of different classes of antimicrobial agents. This ability to resist may be due to some factors such as a lower metabolic rate of the bacterial cell, resistance gene transfer, or even the inability of the antimicrobial agent for permeating into the biofilm. ${ }^{3}$

Polymyxin B has been used on a massive scale in Brazilian hospitals due to the high rate of Klebsiella pneumoniae carbapenemase dissemination. ${ }^{4}$ On the other hand, little is known about what impact on the polymyxin resistance development would have when different forms of bacterial presentation, such as biofilms (which often present in device-related infections), are present.

The aim of this study was to evaluate the impact of biofilm production by $P$. aeruginosa isolates on polymyxin B susceptibility by comparing the minimum biofilm eradication concentration (MBEC) with the minimum inhibitory concentration (MIC). In this 